\title{
Indirekte Nutzung von Computerprogrammen
}

Gianni Fröhlich-Bleuler *

Braucht der Anwender eine Lizenz, wenn er über ein Drittsystem auf ein Computerprogramm zugreift und er es indirekt nutzt? In komplexer werdenden ITSystemlandschaften wird dieser Gebrauch der Software immer wichtiger. Der folgende Beitrag beleuchtet die indirekte Nutzung von Programmen aus urheberrechtlicher Sicht.

I. Einleitung .465

II. Beispiel: Indirekte Nutzung von Computerprogrammen bei Enterprise Resource Planning Software (ERP). 465

III. Betroffene Nutzungsrechte bei der indirekten Nutzung ....................................466

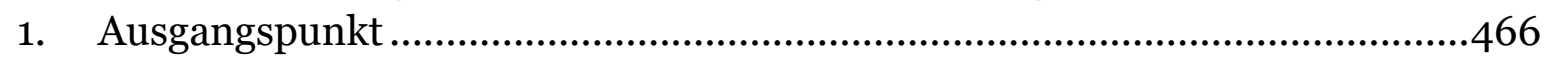

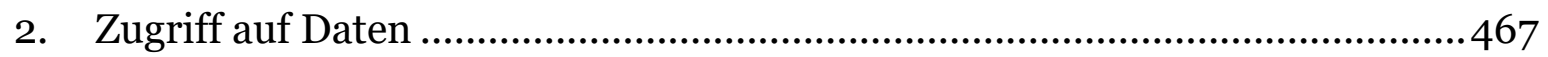

3. Vervielfältigungen ....................................................................................467

4. Öffentliches Zugänglichmachen...................................................................467

IV. Gebrauchs- und Weiterveräusserungsrecht als Schranke? ................................. 471

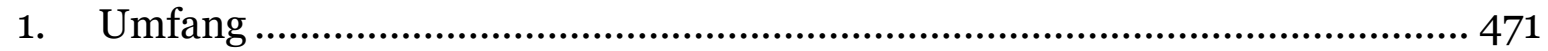

2. Anwendung auf indirekte Nutzung .............................................................4 472

V. Einwilligung des Urhebers (Einräumung Nutzungsbefugnis)..............................472

1. Grundsätzliches ..........................................................................................472

2. Nutzungsbeschränkungen im Vertrag ........................................................... 473

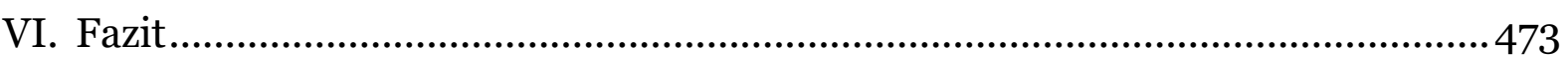

Zitiervorschlag: Gianni Fröhlich-Bleuler, Indirekte Nutzung von Computerprogrammen, in: sui-generis 2018, S. 464

URL: $\quad$ sui-generis.ch/87

DOI: $\quad$ https://doi.org/10.21257/sg.87

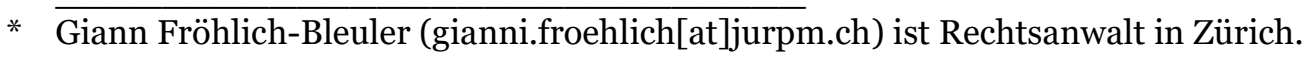




\section{Einleitung}

1 Bei der indirekten Nutzung greift ein Anwender über ein Drittsystem auf ein Computerprogramm zu, das er für diese Nutzung nicht lizenziert hat. Darf er das? Oder braucht er das Einverständnis des Rechteinhabers des Programmes dafür? Die Frage ist wichtig, weil in einer vernetzten Welt immer mehr IT-Systeme miteinander interagieren und indirekt genutzt werden. Im Folgenden gehe ich dieser Frage nach.

2 Ein Computerprogramm ist in der Regel durch das Urheberrecht geschützt. Wann darf ein Anwender es nutzen?

- Erstens, wenn die Nutzung vom Ausschliesslichkeitsrecht des Urhebers gar nicht erfasst ist. In Ziffer III gehe ich daher der Frage nach, ob und allenfalls welche Nutzungsrechte von der indirekten Nutzung betroffen sind.

- Im Urheberrechtsgesetz (URG) sind Schranken definiert. Sie erlauben es dem rechtmässigen Erwerber zweitens, Nutzungsbefugnisse auszuüben, die dem Urheber vorbehalten sind. Die wichtigsten Schrankenbestimmungen sind das Gebrauchs- und Weiterveräusserungsrecht, auf die ich in Ziffer IV näher eingehe.

- Drittens, wenn der Rechteinhaber die Nutzung erlaubt. Die Einwilligung des Rechteinhabers zur indirekten Nutzung ist Thema der Ziffer V.

Zunächst aber gehe ich in Ziffer II auf einige technische Aspekte der indirekten Nutzung ein. Kartell- und AGB-rechtliche Fragen sind nicht Gegenstand dieses Bei- trags. Für ein besseres Verständnis erläutere ich die indirekte Nutzung anhand eines Beispiels, das in Ziffer II beschrieben ist.

\section{Beispiel: Indirekte Nutzung von Computerprogrammen bei Enterprise Resource Planning Software (ERP)}

4 Die meisten Unternehmen setzen Computerprogramme verschiedener Hersteller ein. Die Programme erledigen unterschiedliche Aufgaben. Dafür müssen sie miteinander interagieren. Sie greifen z.B. auf die in anderen Programmen gespeicherten Daten zu oder lösen Funktionen dieser Programme aus. Dazu ein Beispiel: Eine Enterprise Resource Planning Software (ERP) steuert die wichtigsten Prozesse eines Unternehmens. Dazu gehören das Rechnungswesen, die Logistik oder das Personalwesen. Das bekannteste ist «SAP ERP». Ein Programm, das oft mit dem ERP zusammen eingesetzt wird, ist das Customer Relationship Management System (CRM). Mit dem CRM verwalten viele Unternehmen ihre Kundenbeziehungen. Unternehmen, die ein CRM einsetzen, verbinden es mit bestehenden Geschäftsprozessen. Weit verbreitet ist das CRM von Salesforce. Es greift z.B. auf die im ERP gespeicherten Kundendaten $\mathrm{zu}$, wenn ein Kundenbetreuer eine Bestellung für einen Kunden erfasst. Der Bestellprozess wird zwar durch das CRM ausgelöst, die Bestellung selbst aber im ERP abgewickelt. So wird oft etwa der Preis für eine Bestellung zuerst im ERP berechnet und darauf an das CRM bzw. den Kundenberater weitergereicht. Oder die Verfügbarkeit des bestellten Produkts wird über das Logistik-Modul des ERP überprüft. Je nach Konfiguration können 
sogar Kunden des Anwenders das CRM direkt nutzen und eine Bestellung auslösen, die über das ERP ausgeführt wird. Solche Zugriffe werden als «indirekte Nutzung» bezeichnet. Diesem Beispiel liegt der Entscheid «SAP UK Limited vs. Diageo Great Britain Limited» zugrunde. ${ }^{1}$ Darin kommt ein englisches Gericht zum Schluss, dass externe Kunden, die über das CRM von Salesforce auf SAP ERP zugreifen, Nutzer sind, die der Lizenznehmer des ERP lizenzieren muss. ${ }^{2}$

5 Fragen zur indirekten Nutzung stellen sich nicht nur beim Gebrauch eines ERP. In komplexen Systemlandschaften interagieren Computerprogramme auf vielseitige Weise miteinander. Kontrovers diskutiert wird die indirekte Nutzung, weil ihre Auswirkungen sehr unterschiedlich sein können: Prüfen Besucher eines Online-Shops die Verfügbarkeit eines Produktes, lösen sie damit eine Anfrage im ERP aus. Es können damit Tausende von Nutzern sein, die indirekt auf das ERP zugreifen. Muss der Anwender alle diese Nutzer lizenzieren? Anders ist die Situation im folgenden Beispiel: In der Praxis richtet der Anwender oft einen «fiktiven» Nutzer des ERP ein, der korrekt lizenziert ist und über den er alle Zugriffe abwickelt, die im Rahmen der indirekten Nutzung erfolgen. Anstatt alle Nutzer des CRM zu lizenzieren, die indirekt auf das ERP zugreifen, löst der Lizenznehmer nur eine einzige Lizenz. ${ }^{3}$

1 Urteil des Court of Appeal - Technology and Construction Court HT-2015-000340 vom 16. Februar 2017.

2 Die Richterin beurteilte den Fall allerdings nicht unter urheberrechtlichen Aspekten.

3 Sogenanntes «Multiplexing» oder «Pooling» (Metzger Axel/Hoppen Peter, Zur Zulässigkeit von Nutzungsbeschränkungen in Lizenzverträgen bei Verwendung von Drittanbietersoftware, CR 2017, S. 625).
6 Software-Integratoren verbinden das ERP und das CRM auf unterschiedliche Weise miteinander. Sie können auf der Ebene des User Interface, der Daten- oder der Applikationsebene integriert werden. 4 Bei der Integration über das User Interface wird die Benutzeroberfläche eines der beiden Programme im anderen aufgerufen, und die Funktionen des aufgerufenen Programms stehen zur Verfügung. Dabei handelt es sich um einen direkten Zugriff auf das Programm und damit nicht um eine indirekte Nutzung. Diese Form der Nutzung lasse ich hier ausser Acht. Im zweiten Fall tauschen die Programme die Daten aus. Im Fall der Integration auf Applikationsebene schliesslich ruft das eine Programm die Funktionen des anderen über eine Schnittstelle auf (also z.B. das CRM Funktionen des ERP).

\section{Betroffene Nutzungsrechte bei der indirekten Nutzung}

\section{Ausgangspunkt}

7 Gegenstand der Verwertung im Urheberrecht ist das Werk, hier das Computerprogramm. Der Urheber hat das Recht, darüber zu bestimmen, wie das Werk verwendet wird (Art. 10 Abs. 1 URG). Das Urheberrecht erfasst jede Verwertung, bei der die schutzfähigen Teile des Werkes wiedergegeben werden. ${ }^{5}$ Der Umfang des Schutzobjektes definiert damit die Grenzen des Ausschliesslichkeitsrechts

4 Maurer Jürgen, Salesforce-Integration mit dem ERP-System, Computerwoche von 15. April 2015.

5 Dreier Thomas/Schulze Gernot, Urheberrechtsgesetz: UrhG, 6. Aufl., München 2018, Rz. 16 zu $\S 15$. 
des Urhebers. ${ }^{6}$ Meistens prägt die Persönlichkeit des Programmierers die Softwareentwicklung nicht. Das Urheberrecht schützt aber die materiellen Interessen des Rechteinhabers. Der Urheber soll an jeder Verwertung seines Werkes partizipieren. Aus diesem Grund bestimmt in der Regel das Partizipationsinteresse des Urhebers den Umfang der Nutzungsrechte im Softwarebereich. ${ }^{7}$

Im Folgenden behandle ich zuerst den Austausch der Daten zwischen ERP und CRM (Ziffer III.2) und dann die Vervielfältigung des ERP (Ziffer III.3). Beide Formen des Zugriffs stellen keine besonderen urheberrechtlichen Probleme dar. Anders sieht es aus, wenn der Anwender das ERP seinen Nutzern zugänglich macht. Darauf gehe ich daher in der Ziffer III.4 ausführlich ein.

\section{Zugriff auf Daten}

9 ERP und CRM können Daten austauschen. In diesem Fall greift der Nutzer des CRM auf Daten zu, die auch durch das ERP verarbeitet werden. Funktionen im ERP löst er aber keine aus. Es wird

6 Vgl. dazu auch Brändli Sandra, Die Flexibilität urheberrechtlicher Schrankensysteme, Diss., Bern 2017, Rz. 12 sowie Gasser Christoph/Oertli Reinhard, in: Müller Barbara K./Oertli Reinhard (Hrsg.), Handkommentar zum Urheberrecht, 2. Aufl., Bern 2012, Rz. 3 zu Vorbemerkungen zu Art. 19.

7 Vgl. zum Partizipationsinteresse bei der Auslegung des Umfangs der Verwertungsrechte im Softwarebereich exemplarisch Widmer Ursula, Der urheberrechtliche Schutz von Computerprogrammen, Zeitschrift für Schweizerisches Recht (ZSR) 112 I 1993, S. 15 und 19, Rauber Georg, Computersoftware, in: Streuli-Youssef Magda (Hrsg.), Urhebervertragsrecht, Zürich 2006, S. 137 und 146 sowie Straub Wolfgang, Softwareschutz, Zürich 2011, Rz. 127. Straub plädiert für einen Ausgleich der Interessen auf allen Ebenen des Softwareschutzes, also auf der Ebene der Schutzvoraussetzungen, derjenigen des Schutzinhalts oder der zeitlichen Dauer (Straub, Rz. 150). weder vervielfältigt noch sonst wie wiedergegeben. Eine urheberrechtliche Nutzung des ERP erfolgt in diesem Fall nicht. ${ }^{8}$

\section{Vervielfältigungen}

10 Je nach technischer Konfiguration vervielfältigt der Nutzer des CRM beim $\mathrm{Zu}$ griff auf das ERP Teile davon. Sie werden in den Arbeitsspeicher des Nutzers geladen. ${ }^{9}$ Dies ist eine Vervielfältigung und damit eine urheberrechtlich relevante Verwertung des Programmes (Art. 10 Abs. 2 lit. a URG). ${ }^{10}$ In vielen Fällen vervielfältigt der Anwender allerdings das ERP bei der indirekten Nutzung nicht. ${ }^{11}$

\section{4. Öffentliches Zugänglichmachen}

\section{a) Hintergrund}

11 Nur der Urheber hat das Recht, das Werk direkt oder mit Hilfsmitteln anderswo wahrnehmbar oder so zugänglich zu machen, dass Personen von Orten und zu Zeiten ihrer Wahl dazu Zugang haben (Art. 10 Abs. 2 lit. c URG). Findet dieses Verwertungsrecht auf die indirekte Nutzung Anwendung?

12 Art. 10 Abs. 2 lit. c URG beinhaltet das sogenannte On-Demand-Recht gemäss Art. 8 des WIPO-Urheberrechtsvertrages ${ }^{12}$ (WIPO Copyright Treaty/WCT). Der Ge-

8 Metzger/Hoppen (Fn. 3), S. 626; Oelschlägel Kay/Schmidt André, Lizenzpflicht für indirekte Nutzung von SAP-Software, ITRB 2015, S. 73.

9 Das können Teile von Bibliotheken des ERP, Standardroutinen oder Module sein. Dazu ausführlich Metzger/Hoppen (Fn. 3), S. $627 \mathrm{ff}$.

10 Fröhlich-Bleuler Gianni, Softwareverträge, 2. Aufl., Bern 2014, Rz. 1706 (mit Hinweisen zur herrschenden Lehre).

11 Metzger/Hoppen (Fn. 3), S. $628 \mathrm{ff}$.

12 WIPO-Urheberrechtsvertrag, Abgeschlossen in Genf am 20. Dezember 1996 (WCT; SR $0.231 .151)$. 
setzgeber hat die Bestimmung 2008 im Zuge der Umsetzung des WIPOUrheberrechtsvertrages in das URG aufgenommen. Auch die EU hat die Vorgaben dieses Abkommens umgesetzt, und zwar in Art. 3 der Urheberrechtsrichtlinie. ${ }^{13}$ Im Schweizer Legislativprozess wurde betont, dass sich die Anpassung in Art. 10 Abs. 2 lit. c URG an die EUGesetzgebung anlehne. ${ }^{14}$

13 Für eine öffentliche Wiedergabe gemäss Art. 10 Abs. 2 lit. c URG müssen drei Voraussetzungen gegeben sein:15 Erstens macht der Anwender das Programm zugänglich (Ziffer III.4.b), und zweitens der Öffentlichkeit (Ziffer III.4.c); und schliesslich erfolgt der Zugang drittens von einem Ort und zu einem Zeitpunkt nach Wahl des Nutzers (Ziffer III.4.d). Im Folgenden gehe ich auf diese drei Voraussetzungen ein.

\section{b) Zugang zum Programm}

14 Als erste Voraussetzung muss der Anwender einem Dritten Zugriff auf das Computerprogramm oder einen urheberrechtlich geschützten Teil davon geben. Art. 10 Abs. 2 lit. c URG verwendet dafür in der deutschen Fassung den Begriff «Zugang» zum Werk. ${ }^{16}$ Der Tatbestand wird meines Erachtens erfüllt, wenn der Nutzer Zugang zu Funktionen des Programmes erhält. Es ist daher nicht nötig, dass der urheberrechtlich geschützte

13 Richtlinie 2001/29/EG zur Harmonisierung bestimmter Aspekte des Urheberrechts und der verwandten Schutzrechte in der Informationsgesellschaft.

14 Vgl. z.B. Votum Müller Thomas, AB 2007, N. 1198; Votum Bundesrat Blocher Christoph, AB 2007, N. 1202.

15 Dreier/Schulze (Fn. 5), Rz. 6 ff. zu § 19a.

16 In der französischsprachigen Fassung wird dafür «accès» und der italienischen "accedervi» verwendet; Art. 8 WCT spricht von «making available».
Code des Programmes selbst für den Nutzer zugänglich ist. ${ }^{17}$ Dies ergibt sich erstens daraus, dass sowohl im WCT als auch im URG der Begriff «Zugang» technologieneutral auszulegen ist. ${ }^{18}$ Es spielt daher keine Rolle, wie der Nutzer auf das Programm zugreift und ob der Code selber zugänglich ist. Zweitens spricht das Partizipationsinteresse des Urhebers für dieses Verständnis. Durch das Zugänglichmachen der Funktionalität - und nicht durch den Zugang zum Code - erschliesst sich der Anbieter einen zusätzlichen Nutzerkreis. Im oben genannten Beispiel sind dies einerseits die zusätzlichen Mitarbeiter des Anwenders und andererseits seine Kunden. Beide können die Funktionen des ERP nutzen, ohne selbst als Nutzer lizenziert zu sein, weil sie über das CRM auf das ERP zugreifen.

15 Wie weit geht der lizenzpflichtige $\mathrm{Zu}-$ griff? Ein ERP läuft in einer Systemumgebung mit verschiedenen Programmen. Beim Abruf von Funktionen des ERP werden auch Programme aus der Systemumgebung verwendet. Dazu gehören z.B. das Betriebssystem, auf dem das ERP läuft, die Datenbank oder die Middleware, die die Interoperabilität zwischen den Programmen ermöglicht. Werden al-

17 Dreier/Schulze (Fn. 5), Rz. 36 ff zu § 69c; Spindler Gerald, Rz. 67 zu Vor $\S \S 69$ a ff., in Schricker Gerhard/Loewenheim Ulrich (Hrsg.), Urheberrecht, 5. Aufl., München 2017; a.M. Straub, Rz. 133; Grützmacher Malte, in: Wandtke ArturAxel/Bullinger Winfried, 4. Aufl., München 2010, Rz. $66 \mathrm{zu} \S 69 \mathrm{c}$ UrhG.

18 Für das WCT: Von Lewinski Silke, The WIPO Treaties on Copyright - A Commentary on the WCT, the WPPT, and the BTAP, 2. Aufl., Oxford 2015, Rz. 7.8.32 zu Article 8. Generell zur Technikneutralität des URG: BGE 140 III 616 E. 3.4.1; Barrelet Denis/Egloff Willi, Das neue Urheberrecht, 3. Aufl. 2008, Rz. 7a zu Art. 10 URG; Brändli (Fn. 6), Rz. 116; vgl. zur Technikneutralität des «Zugänglichmachens» auch Dreier/ Schulze (Fn. 5), Rz. 6 zu § 19a. 
le diese Programme beim Abruf einer Funktion des ERP zugänglich gemacht und damit im Sinne des Urheberrechts verwertet?

Ausgangspunkt für die Beantwortung dieser Frage ist wiederum das Partizipationsinteresse des Software-Herstellers. Es stellt sicher, dass der Urheber von jeder urheberrechtlichen Nutzung des Programms profitieren kann. Die übliche Verwertung des zugänglich gemachten Programmes (oder des entsprechenden Teiles) darf durch die indirekte Nutzung nicht beeinträchtigt werden. Mit anderen Worten darf es durch die indirekte Nutzung nicht substituiert werden. ${ }^{19}$ Wie kann dies festgestellt werden? Die Mikroökonomie unterscheidet zwischen Substitutions- und Komplementärgütern. ${ }^{20}$ Güter, die dieselben oder ähnliche Bedürfnisse stillen und sich deshalb gegenseitig ersetzen können, sind Substitutionsgüter. Das sind z.B. CD und Streaming im Musikmarkt. ${ }^{21}$ Demgegenüber sind Güter, die gemeinsam nachgefragt werden, weil sie sich in ihrem Nutzen ergänzen, Komplementärgüter. ${ }^{22}$ Dazu gehören z.B. Hammer und Nagel. ${ }^{23}$ Im oben genannten Beispiel sind das CRM und das ERP im Hinblick auf die Bestellfunktion Substitutionsgüter. Sie decken diesbezüglich die gleichen Bedürfnisse ab. Daher wird das Partizipationsinteresse des Herstellers des ERP verletzt, wenn ein Mitarbeiter oder ein Kunde des Anwenders über die Bestellfunktion des CRM die Bestellung im ERP abwickelt.

17 Anders ist es aber bei Programmen, die bezüglich der indirekt genutzten Funktionen keine Substitutionsgüter sind. Dies gilt z.B. für das Betriebssystem. Löst der Nutzer des CRM die Bestellfunktion aus, werden keine Funktionen im Betriebssystem angestossen, die der Bestellfunktion entsprechen und sie substituieren. Mit anderen Worten ergänzen die Funktionen des Betriebssystems diejenigen des CRM - CRM und Betriebssystem sind keine Substitutionsgüter. Damit wird das Verwertungspotenzial des Betriebssystems bei der indirekten Nutzung durch das CRM nicht tangiert. Im oben genannten Beispiel umfasst die indirekte Nutzung damit den Gebrauch des Betriebssystems nicht. ${ }^{24}$ Diese Überlegung gilt auch für die Datenbanksoftware: Sie ist kein Substitutionsgut zum CRM und ihre indirekte Nutzung wird daher nicht von Art. 10 Abs. 2 lit. c URG erfasst.
$19 \overline{\text { Vgl. dazu Gasser/Oertli (Fn. 6), Rz. } 8 \text { zu Vorbe- }}$ merkungen zu Art. 19.

20 Vgl. dazu Landes William M./Posner Richard A., The Economic Structure of Intellectual Property Law, Cambridge 2003, S. 89, die den Test dafür verwenden, urheberrechtlich relevante Kopien von der freien Nutzung zu unterscheiden.

21 In der Microökonomie werden zwei Güter als vollkommene Substitutionsgüter betrachtet, wenn die Grenzrate der Substitution des einen durch das andere Gut eine Konstante ist (Pindyck Robert S./Rubinfeld Daniel L., Mikroökonomie, 8. Aufl., München 2015, S. 118).

22 Seite Wikipedia, «Komplementärgut».

23 Landes/Posner (Fn. 20), S. 154.
24 Meyer Oliver, Aktuelle vertrags- und urheberrechtliche Aspekte der Erstellung, des Vertriebs und der Nutzung von Software, Diss., Karlsruhe 2008, S. 303 und S. 325 (allerdings mit anderer Begründung); a.M. Koch, Grid Computing im Spiegel des Telemedien-, Urheber- und Datenschutzrechts, Computer und Recht 2006, S. 117; Giedke Anna, Cloud Computing: Eine wirtschaftsrechtliche Analyse mit besonderer Berücksichtigung des Urheberrechts, Diss., München 2013, S. 404. 
Die gleiche Wertung liegt Art. 11 des TRIPS-Abkommens ${ }^{25}$ zugrunde. Die Mitgliedstaaten des Abkommens müssen sicherstellen, dass der Urheber darüber entscheiden kann, ob sein Werk vermietet wird. Diese Verpflichtung gilt bei einem Computerprogramm allerdings nur, wenn es der wesentliche Gegenstand der Vermietung ist. Sie findet z.B. auf die Vermietung eines Autos keine Anwendung, in dem Computerprogramme installiert sind. Der Vermieter des Wagens darf die Programme mitvermieten, und zwar ohne das Einverständnis des Urhebers der Programme. Dies gilt allerdings nur, wenn das Auto das wesentliche Objekt der Vermietung ist. Begründen lässt sich die Ausnahme auch hier wie folgt: Die im vermieteten Fahrzeug installierte Software ist kein Substitutionsgut für die Programme. ${ }^{26}$

\section{c) Öffentlichkeit}

19

Das Computerprogramm wird der Öffentlichkeit zugänglich gemacht. Dies ist die zweite Voraussetzung für die Anwendung von Art. 10 Abs. 2 lit. c URG. In diesem Fall kann eine grössere Anzahl von Nutzern ausserhalb eines Kreises von Personen, die eng miteinander verbunden sind, darauf zugreifen (Art. 9 Abs. 3 URG). Entscheidend ist die Anzahl der Personen. ${ }^{27}$ Es genügt, dass sie $\mathrm{Zu}-$ gang zum Programm haben und von ihm Kenntnis nehmen können. ${ }^{28}$ Allerdings liegt «Öffentlichkeit» nur vor, wenn mehr als eine Person Zugriff hat.

20 Sobald ein grösseres Unternehmen Mitarbeitern den Zugang zu einem Programm ermöglicht, ist es öffentlich zugänglich. ${ }^{29}$ Sie sind in der Regel nicht eng miteinander verbunden. In unserem Beispiel wird das ERP damit sowohl für die Mitarbeiter des Anwenders, die über das CRM darauf zugreifen, als auch für die Kunden des Anwenders öffentlich zugänglich.

\section{d) Zugang von Ort und Zeitpunkt nach Wahl des Nutzers}

21 Schliesslich setzt drittens das Recht auf Zugänglichmachung voraus, dass die Nutzer von einem Ort und zu einem Zeitpunkt ihrer Wahl auf das Computerprogramm zugreifen können. Diese Voraussetzung ist für die Mitarbeiter und die Kunden des Anwenders ebenfalls gegeben.

\section{e) Zwischenfazit}

22 Im eingangs genannten Beispiel greift der Anwender bei einer indirekten Nutzung in zwei Fällen in das Verwertungsrecht des Urhebers ein: Erstens bei einer Vervielfältigung des ERP, zweitens, wenn

27 Neff Emil/Arn Matthias, Urheberrechtlicher Schutz der Software, in: von Büren Roland/David Lucas (Hrsg.): Schweizerisches Immaterialgüter und Wettbewerbsrecht, Bd. II/2, Basel/Frankfurt a.M. 1998, S. 210.

28 Neff/Arn (Fn. 27), S. 210.

29 Fröhlich-Bleuler Gianni, Urheber- und vertragsrechtliche Aspekte der Open Source Software, in: Jörg Florian S./Arter Oliver (Hrsg.), IT-Verträge, Bern 2007, S. 212; Dreier/Schulze (Fn. 5), Rz. 7 zu § 19a (Intranet). 
ein Mitarbeiter oder ein Kunde des Anwenders über das CRM auf Funktionen des ERP zugreift und diese Funktionen durch das CRM substituiert werden.30 Der Mitarbeiter oder der Kunde sind dazu aber befugt, wenn die Nutzung durch eine Schrankenbestimmung gedeckt ist oder wenn der Software-Hersteller sein Einverständnis dazu gegeben hat. Auf diese beiden Punkte gehe ich im Folgenden ein.

\section{Gebrauchs- und Weiterveräusse- rungsrecht als Schranke?}

\section{Umfang}

23 Die im URG vorgesehenen Schrankenbestimmungen erlauben es dem Anwender, Nutzungsbefugnisse auszuüben, die sonst dem Urheber vorbehalten sind. Die wichtigste EDV-rechtliche Schranke im Urheberrecht ist das Gebrauchs- und Weiterveräusserungsrecht. Gemäss Art. 12 Abs. 2 URG darf der Erwerber des Werkexemplars dieses gebrauchen oder weiterveräussern (Erschöpfungsprinzip). Damit wird sichergestellt, dass ein Werkstück frei von urheberrechtlichen Bindungen gehandelt werden kann. Zudem hat der Urheber bei der ersten Verbrei-

30 A.M. ein Teil der Literatur in Deutschland: Sie taxiert die indirekte Nutzung - insbesondere im Fall von SAP ERP - nicht als öffentliche Wiedergabe. Sie prüft die indirekte Nutzung im Wesentlichen nur unter dem Aspekt, ob eine nicht erlaubte Vervielfältigung durch den Nutzer vorliegt, was in der Regel nicht der Fall ist (Metzger/Hoppen [Fn. 3], S. 633; Schneider Jochen, in: Schneider Jochen [Hrsg.], Handbuch EDVRecht, 5. Aufl., Köln 2017, Rz. 332 ff. zu G.; Barnizke Benno, Zur indirekten Nutzung von Software - Komplex, intransparent und unwirksam? K\&R 2018, S. 457). Anders demgegenüber die deutsche Literatur, die die indirekte Nutzung im Zusammenhang mit Application Service Providing oder Cloud Computing behandelt (siehe dazu die Nachweise in Fn. 24). tung das ihm zustehende Entgelt erhalten. Er hat kein berechtigtes Interesse, an weiteren Verbreitungshandlungen $\mathrm{zu}$ partizipieren. ${ }^{31}$ Art. 12 Abs. 2 URG beschränkt damit das Verwertungsrecht des Urhebers, während es den Anwender berechtigt, das Programm ohne das Einverständnis des Urhebers bestimmungsgemäss zu nutzen.

24 Der Umfang des Gebrauchsrecht ergibt sich aus dem Vertrag zwischen dem Urheber und dem Anwender, der Art des Programmes, den Funktionen oder dem Zweck, für das es überlassen wurde. Der Anwender darf daher das Programm so gebrauchen, wie es für die vereinbarte Nutzung erforderlich ist. ${ }^{2}$ Haben die Parteien keine Vereinbarung zur Nutzung getroffen, finden die gesetzlichen Bestimmungen von Art. 17 Abs. 1 lit. a URV als dispositives Recht Anwendung.33 Das Gebrauchsrecht beinhaltet aber einzig ein auf den Gebrauch beschränktes Vervielfältigungs- und ein limitiertes Bearbeitungs- und Fehlerbehebungsrecht. Es umfasst das in Art. 10 Abs. 1 lit. c URG genannte Recht zum öffentlichen Zugänglichmachen des Computerprogrammes nicht. 34

${ }_{31} \overline{\text { Fröhlich-Bleuler Gianni, Urheberrechtliche Nut- }}$ zungsbefugnisse des EDV-Anwenders, Aktuelle Juristische Praxis (AJP) 6/1995, S. 570.

32 Fröhlich-Bleuler (Fn. 10), Rz. 154 sowie 156.

33 Gemäss Straub handelt es sich dabei um den Mindestinhalt von Befugnissen, die dem Erwerber der Programmkopie zustehen (Straub [Fn. 7], Rz. 177).

34 Dreier/Schulze (Fn. 5), Rz. 10 zu § 69d; FröhlichBleuler (Fn. 10), Rz. 158 ff.; Kotthoff Jost, Rz. 3 zu § 69d, in: Dreyer Gunda/Kotthoff Jost/Meckel Astrid (Hrsg.), Urheberrecht, 2. Aufl., Heidelberg 2008; a.M. Straub (Fn. 7), Rz. 170 sowie wohl Grützmacher für unternehmensinternen öffentlichen Zugang (Grützmacher [Fn. 17], Rz. 13 zu $\S 69 d)$. 
Das Gebrauchsrecht enthält einen zwingenden Kern, von dem die Parteien im Vertrag nicht abweichen dürfen. Dazu gehört z.B. das Recht, das Programm zu installieren - sofern dies für die Nutzung notwendig ist - und die für den Gebrauch notwendigen Vervielfältigungen vorzunehmen. 35

\section{Anwendung auf indirekte Nutzung}

\section{a) Indirekte Nutzung mit Vervielfältigung des ERP}

26 Kopiert der Nutzer das ERP bei der indirekten Nutzung, so kann dies vom bestimmungsgemässen Gebrauch gedeckt sein. Dies gilt z.B., wenn der Hersteller das Programm bei der Auslieferung bereits für die indirekte Nutzung vorbereitet hat. In diesem Fall ist der Anwender befugt, das ERP im dafür notwendigen Umfang zu kopieren.

\section{b) Indirekte Nutzung bei öffentlichem Zugänglichmachen}

Wie oben ausgeführt, umfasst das Gebrauchsrecht das öffentliche Zugänglichmachen gemäss Art. 10 Abs. 1 lit. c URG nicht. Für die indirekte Nutzung kann sich der Anwender daher nicht auf das Recht zum bestimmungsgemässen Gebrauch berufen. Dafür braucht der Anwender die Zustimmung des Rechteinhabers, die ich in der folgenden Ziffer behandle.

\section{Einwilligung des Urhebers (Einräumung Nutzungsbefugnis)}

\section{Grundsätzliches}

28 Der Anwender darf das Programm nutzen, wenn er das Einverständnis des Rechteinhabers hat. Dies ist die dritte Möglichkeit, wie er die indirekte Nutzung rechtfertigen kann. Art und Umfang der eingeräumten Nutzungsbefugnisse richten sich wie bei anderen Verträgen nach dem Verpflichtungsgeschäft. Der Hersteller kann die Einwilligung ausdrücklich einräumen, z.B. indem die Parteien die Nutzungsbefugnis im Vertrag regeln. Ist die Vereinbarung unklar, muss sie ausgelegt werden. Nicht die einzige, aber die wichtigste Auslegungsregel ist die Zweckübertragungstheorie. Für die Bestimmung des Umfangs der eingeräumten Nutzungsbefugnisse ist der Vertragszweck massgeblich. ${ }^{6}$ Oft ergibt er sich aus den Funktionen des lizenzierten Softwareprogramms, wenn sie im Vertrag vereinbart sind. Oder er resultiert aus den im Vertrag beschriebenen Umsystemen. Der Vertragszweck kann sich aber auch aus den vereinbarten Leistungen des Herstellers ergeben. Verpflichtet sich der Hersteller z.B., das gelieferte ERP über eine Schnittstelle an den Online-Shop anzubinden, gehört zum Vertragszweck auch die indirekte Nutzung über den Onlineshop.
35 Vgl. dazu und für weitere Nutzungsbefugnisse Fröhlich-Bleuler (Fn. 10), Rz. 163 f.
36 Barrelet/Egloff (Fn. 18), Rz. 20 zu Art. 16; Laux Christian, Vertragsauslegung im Urheberrecht, Diss., Bern 2003, S. 83 f.; vom vertragsgemässen Gebrauch des Computerprogramms ist der oben genannte bestimmungsgemässe Gebrauch zu unterscheiden, der sich - teilweise - aber ebenfalls aus dem Überlassungsvertrag ergibt (siehe dazu Fröhlich-Bleuler (Fn. 10), Rz. 154 ff.). 
2. Nutzungsbeschränkungen im Vertrag

29 Es steht den Parteien allerdings frei, das Vervielfältigungsrecht oder das Recht auf Zugänglichmachen zu beschränken, zum Beispiel auf eine bestimmte Anzahl von Nutzern, die auf das Programm direkt oder indirekt zugreifen. Dabei haben die Parteien den zwingenden Kern des Gebrauchsrechts zu beachten. 37

3 So machte bis vor kurzem SAP in ihren Vertragsbedingungen die Höhe der Lizenzgebühr für die Nutzung des ERP von der Anzahl «Named User» abhängig. ${ }^{8}$ Named User sind registrierte Nutzer. Sie werden unabhängig davon, ob sie zu einem bestimmten Zeitpunkt auf das lizenzierte Programm zugreifen, für die Berechnung der Lizenzgebühr mitgezählt. 39 Ausdrücklich bestimmte SAP auch, dass die indirekte der direkten Nutzung gleichgestellt sei. Im eingangs erwähnten englischen Urteil führte dies dazu, dass die Richterin die Mitarbeiter und die Kunden des Anwenders als lizenzpflichtige Nutzer wertete; dementsprechend musste der Anwender SAP nachträglich Lizenzgebühren von über 54 Millionen Pfund nachzahlen. 40

\section{Fazit}

31 Sofern der Anwender das indirekt genutzte Programm vervielfältigt oder er es Mitarbeitern oder Dritten zugänglich macht, greift er in das Urheberrecht des Herstellers der Software ein. Beschränkt sich die indirekte Nutzung auf den Austausch der Daten, gilt dies nicht. Zur Vervielfältigung kann der Anwender je nach den Umständen aufgrund des gesetzlichen Gebrauchsrechts jedoch befugt sein, nicht aber zum öffentlichen $\mathrm{Zu}-$ gänglichmachen. Manchmal ergibt sich aber aus dem Zweck des abgeschlossenen Vertrages, dass der Hersteller dem Anwender die indirekte Nutzung erlaubt hat.

\footnotetext{
Vgl. dazu oben Ziffer IV.1.

38 In der Zwischenzeit hat SAP die Nutzungsbedingungen angepasst. Die Anwender können wählen, ob sie das alte Lizenzregime beibehalten oder zum neuen wechseln. Letzteres unterscheidet zwischen «Human Access» und «Digital Access», das die indirekte Nutzung umfasst (Söbbing Thomas, Neue SAP-Lizenzbedingungen, ITRB 2018, S. 161).

39 Fröhlich-Bleuler (Fn. 10), Rz. 1759.

40 Urteil des Court of Appeal - Technology and Construction Court HT-2015-000340 vom 16. Februar 2017, Rz. 8.
} 